\title{
$X$-linked juvenile retinoschisis in a black South African: A case report
}

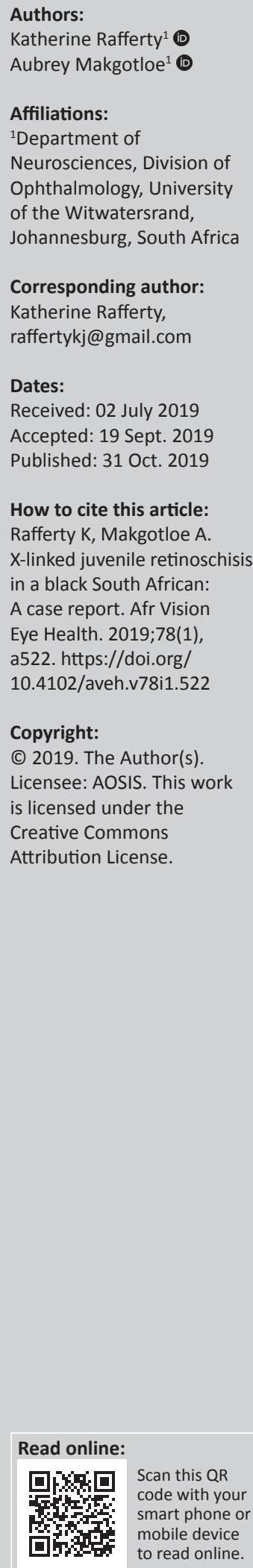

X-linked juvenile retinoschisis (XLRS) has been well-documented in Finnish and other white populations. This case report illustrates a well-recognised condition in a rare population group. There are few case reports in literature of XLRS in black populations.

Keywords: foveal retinoschisis; X-linked juvenile retinoschisis; macular schisis; black patient; South African.

\section{Introduction}

X-linked juvenile retinoschisis (XLRS) is an X-linked recessive bilateral retinal dystrophy that affects young males in their first decade of life. . $^{1,2,3,4,5,6}$ It is the leading cause of juvenile macular degeneration in males with a prevalence estimated between 1 in 15000 and 1 in $30000 .^{1,4}$ The implicated gene is the RS1 gene (Xp22.1), which codes for the adhesion protein retinoschisin. . $^{1,3,4,6}$ More than 190 different genetic mutations have been documented, resulting in a highly variable clinical expression. ${ }^{3}$ Even patients with the same underlying genetic mutation in the same family can display differences in both severity and progression. ${ }^{3,4}$

Patients typically present with poor vision or difficulty in reading in the first decade of life. ${ }^{1,3,4,6,7}$ Refraction is more likely to reveal a hyperopic script. The initial drop in visual acuity is typically followed by a long stable period, up until the fifth or sixth decade of life where atrophic macular degeneration then results in further deterioration of vision. ${ }^{5}$ It is important to note that the clinical presentation is variable, and the visual acuity of an affected child can reveal anything from mild to severe impairment $(6 / 12$ to $6 / 60) \cdot{ }^{1,3,4,5}$

Clinically symmetrical foveal schisis (splitting of the retina) $)^{3,4}$ is characteristic and seen in $98 \%-$ $100 \%$ of cases. ${ }^{4,7}$ This results in a bilateral spoke-wheel pattern of folds that radiates out from the foveola. ${ }^{1,3,4,5}$ Vitreous veils with elevation of the surface retinal layer are commonly observed, $, 1,3,5,7$ and approximately half of the patients have an accompanying peripheral retinoschisis which is usually inferotemporal in location. . $^{1,3,4,5,7,8}$

Optical coherence tomography (OCT) has become an important diagnostic examination ${ }^{3}$ showing splitting and cystic changes of inner nuclear, outer plexiform and outer nuclear layers, ${ }^{1,4}$ a shift from the earlier histopathological studies showing nerve fibre layer separation. Fundus fluorescein is essentially normal and allows one to exclude other differentials. ${ }^{1}$ Dark-adapted electroretinogram (ERG) testing classically reveals a selective reduction in the b-wave amplitude with preservation of the negative a-wave, ${ }^{3,4,5}$ but it is important to be aware that variability is a feature and a normal ERG can also be seen. ${ }^{1,4,5}$

The differential diagnosis for bilateral foveal schisis includes conditions that cause cystic macular changes, with the emphasis on those that do not cause leakage on fundus fluorescein angiography (FFA). These include inherited conditions such as X-linked congenital stationary night blindness (XLCSNB) and Goldmann-Favre vitreoretinal degeneration (GFVD). ${ }^{4,5,7}$ These typically present with early onset nyctalopia, which is not a feature of XLRS. Drugs such as niacin that cause a reversible cystic maculopathy need to be excluded. ${ }^{1,2}$ Optic nerve pits and myopic macular schisis as well as other structural changes at the macula (epiretinal membrane, vitreomacular traction) should also be excluded with a careful clinical examination. ${ }^{1,2}$ In patients who present with the disorder later in life, degenerative retinoschisis can also be considered. ${ }^{1}$

A recently described foveomacular retinoschisis called stellate non-hereditary idiopathic foveomacular retinoschisis can have a clinical presentation almost identical to XLRS, but this has 
been described in females and has unilateral involvement, and genetic testing is negative for the RS1 gene. ${ }^{2}$

In terms of management, this is focused on potential complications. ${ }^{1}$ Vitreous haemorrhage and retinal detachment can occur, ${ }^{1,4,7}$ making follow-up of these patients important for early identification and management, as well as to avoid amblyopic consequences. ${ }^{5}$ Despite these potential complications, patients usually have stable visual acuity until they are 50-60 years of age, when a decline can be noted secondary to atrophic macular changes. ${ }^{1}$ With regards to treatment, carbonic anhydrase inhibitors are of limited value, but with advances in genetic therapies, especially with the current interest in adeno-associated viral vectors, more definitive therapy options will hopefully become available..$^{3,4,5}$

\section{Case report}

Mr T.M. is a 23-year-old black South African male with a history of mild myopia, who presented at age 19 with the main complaint of bilateral gradual loss of vision. He did not have a notable history of nyctalopia nor a history of trauma or chronic medication use. He had no significant family history of a loss of vision. He has a 2-year-old daughter, who has no ocular complaints. There is also no known mixed-ancestry in his family. He works as a supervisor at a merchandising company and is a non-smoker and social drinker. On examination, his best-corrected Snellen visual acuity was $6 / 12$ bilaterally, with a subjective refraction of: Plano/ -1.75 axis $90^{\circ}$ for the right eye and $-0.25 /-1.75$ axis $84^{\circ}$ for the left eye. On slit-lamp biomicroscopy, his anterior segment examination was normal; his intraocular pressures were $12 \mathrm{~mm} \mathrm{Hg}$ and $13 \mathrm{~mm} \mathrm{Hg}$ for the right and left eye respectively. His lenses were clear bilaterally. He had bilateral syneretic vitreous and on fundoscopy he exhibited a bilateral, symmetrical spoke-like pattern at the macula. His optic nerves were normal with a cup-to-disc ratio of 0.3 and no optic nerve pit was noted. No peripheral retinal abnormalities were seen; there were no features of a myopic fundus and no pigmentary changes were noted. No peripheral retinoschisis was evident.

Spectral domain-optical coherence tomography (SD-OCT) confirmed a bilateral, symmetrical foveal schisis (see Figure 1). Fundus autofluorescence (FAF) imaging and FFA highlighted the symmetrical 'spoke-like' macular pattern and revealed no active leakage, with some mild intraretinal pooling (Figures 1 and 2).

Electrophysiological tests revealed a diminished b-wave (Figure 3). The patient has been referred for genetic counselling and testing.
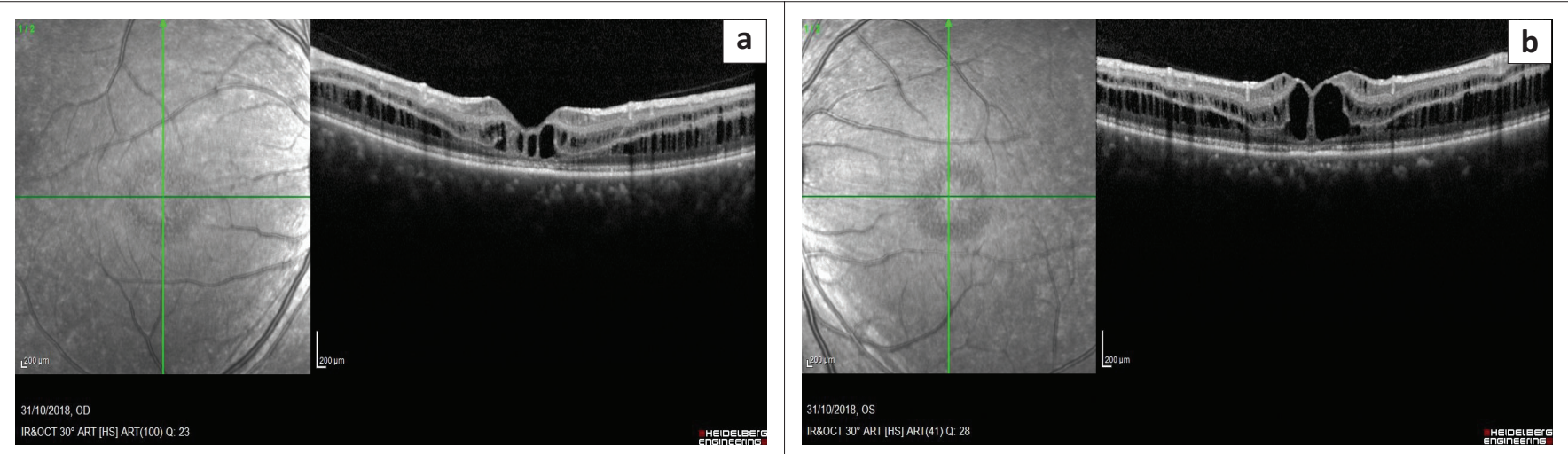

Source: Photo courtesy of Dr Tebogo Maleka.

FIGURE 1: Spectral domain-optical coherence tomography of the right (a) and left eye (b) respectively. There are splitting/cystic changes in the inner nuclear layer (INL), the outer plexiform layer (OPL) and the outer nuclear layer (ONL). A finding consistent with XLRS cases in current literature (Schofield et al. 2016) ${ }^{1}$ Our patient also presents with retinoschisis beyond the clinical impression and spoke-wheel pattern - a feature reported in other cases in the literature.
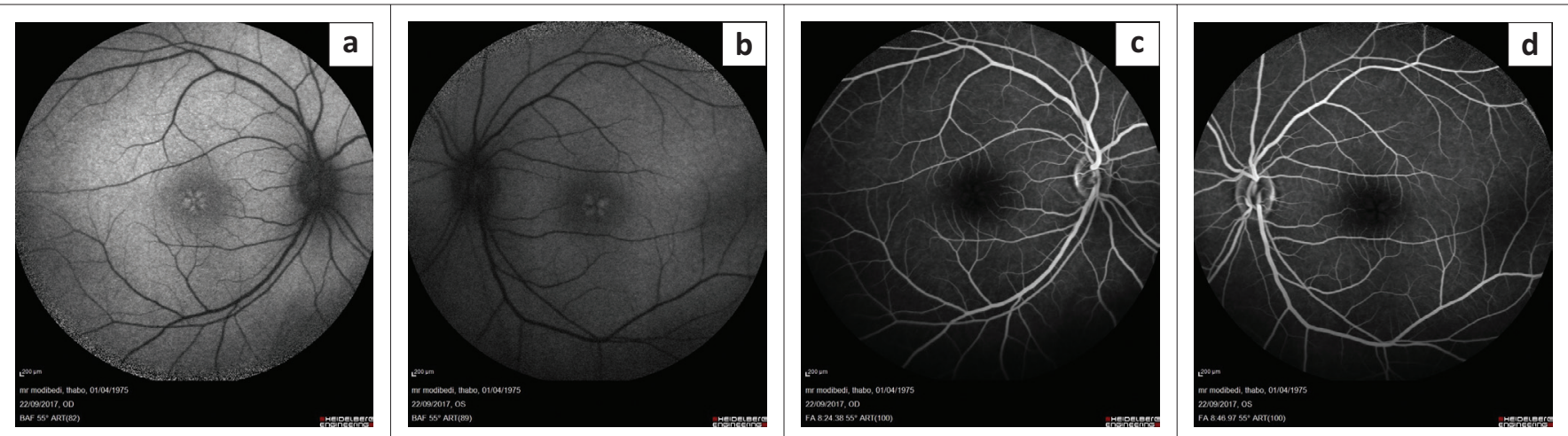

Source: Photo courtesy of Dr Tebogo Maleka.

FIGURE 2: ( $a, b)$ Fundus autofluorescence (FAF) and (c, d) fundus fluorescein angiography (FFA) images of the right and left eye respectively. In the fundus autofluorescence images the 'spoke-like' pattern is clearly highlighted. The fundus fluorescein angiography shows no active leakage, with mild pooling at the macula. 


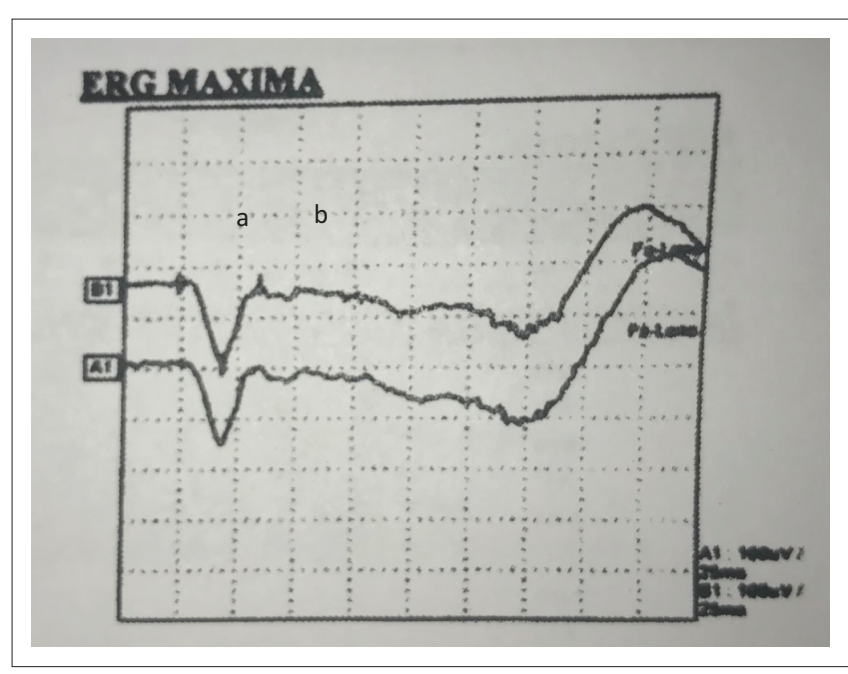

Source: Steve Biko Academic Hospital Neurophysiology Laboratory.

FIGURE 3: Electroretinogram (ERG) showing a selective reduction in b-wave amplitude.

\section{Ethical considerations}

Ethical clearance to conduct this study was obtained from the Human Research Ethics Committee (medical), University of the Witwatersrand, Johannesburg (ethical clearance number: M190699).

\section{Discussion}

When considering the rare diagnosis of XLRS in this case, as mentioned previously, there are certain differentials one needs to exclude in a patient presenting with bilateral, symmetrical foveal schisis. Macular schisis associated with myopia is a more common, similar presentation. It is thought that after a posterior vitreous detachment, residual cortex can cause tangential traction on the macula, and in association with anterior-posterior forces of a staphyloma, macular schisis occurs. ${ }^{1,2}$ With only a mildly myopic script and no other myopic fundus features (e.g. tilted disc, peripapillary atrophy and associated posterior staphyloma), this diagnosis was excluded. Optic nerve pits are also more common but none were seen in this case. Retinal cystic changes with no leakage on fluorescein angiography (FA) was a strong diagnostic point. X-linked congenital stationary night blindness, some forms of retinitis pigmentosa and GFVD can be seen with this isolated finding, but, given that there is no classic early onset of nyctalopia, these diagnoses are unlikely.,5,7 Drug toxicity is also a possibility (e.g. Niacin) ${ }^{1}$ but with no medications elicited, also unlikely. Other rare genetic conditions such as autosomal recessive and autosomal dominant retinoschisis have been described, but these tend to have normal ERG findings. ${ }^{4,5,7}$

X-linked juvenile retinoschisis (XLRS) is well-documented in the literature. The highest prevalence is in Finland. ${ }^{4,7,8}$ Although it has been described in black patients, ${ }^{8,9,10}$ the paucity of documented case reports in the literature of black patients presenting with XLRS prompted this case report. To our knowledge, there have only been three case reports of black patients with XLRS (two family reviews conducted in the 1970s) $)^{8,10}$ and a recent case report in a Nigerian child. ${ }^{8}$ The first was published in 1972 by Constantaras et al. who described XLRS in an African-American family. ${ }^{9}$ The second, more recent article is a case report published in the Nigerian Journal of Ophthalmology in 2018. ${ }^{8}$ This case report is of a 12-year-old male who presented with foveal schisis as well as inferotemporal schisis that was not seen in our case.

Hypermetropia is usually an association, although this association was not found in our patient or the Nigerian case report.

\section{Acknowledgements}

The authors would like to thank Dr Tebogo Maleka for his assistance with the imaging of the patient. They also thank the Steve Biko Academic Hospital Neurophysiology Laboratory for performing the electroretinogram on the patient.

\section{Competing interests}

The authors have declared that no competing interests exist.

\section{Authors' contributions}

Both authors contributed equally to this work.

\section{Funding information}

This research received no specific grant from any funding agency in the public, commercial or not-for-profit sectors.

\section{Data availability statement}

Data sharing is not applicable to this article as no new data were created or analysed in this study.

\section{Disclaimer}

The views and opinions expressed in this article are those of the authors and do not necessarily reflect the official policy or position of any affiliated agency of the authors.

\section{References}

1. Scofield S, Chen R. Chapter 79: Macular retinoschisis. In: Medina C, Townsend J, Singh A, editors. Manual of retinal diseases: A guide to diagnosis and management. Switzerland: Springer, 2016; pp. 397-400.

2. Ober $M$, Freund $K$, Shah $M$, et al. Stellate nonhereditary idiopathic foveomacular retinoschisis. Ophthalmology. 2014;121(7):1406-1413. https://doi.org/10.1016/ j.ophtha.2014.02.002

3. Molday R, Kellner U, Weber B. X-linked juvenile retinoschisis: Clinical diagnosis, genetic analysis, and molecular mechanisms. Prog Retin Eye Res. 2012;31(3): 195-212. https://doi.org/10.1016/j.preteyeres.2011.12.002

4. Sikkink S, Biswas S, Parry N, et al. X-linked retinoschisis: An update. J Med Genet. 2007;44(4):225-232. https://doi.org/10.1136/jmg.2006.047340

5. Sieving P, MacDonald I, Chan S. X-linked juvenile retinoschisis. GeneReviews. Seattle, WA: National Library of Medicine, National Institute of Health, University of Washington; 2014 [cited 2017 Nov 21]. Available from: https://www.ncbi.nlm. nih.gov/books/NBK1222/.

6. American Academy of Ophthalmology. Retina/vitreous: Juvenile retinoschisis. Practicing ophthalmologists learning system 2017-2019. San Francisco, CA: American Academy of Ophthalmology; 2017. 
7. George N, Yates J, Moore A. X-linked retinoschisis. Br J Ophthalmol. 1995;79(7): 697-702. https://doi.org/10.1136/bjo.79.7.697

8. Guirou N, Sylla F, Abba Kaka YH, Thera J, Bakayoko S, Dougnon A, et al. X-linked juvenile retinoschisis: A case report. Niger J Ophthalmol. 2018;26(1):82-84. https://doi.org/10.4103/njo.njo_28_17
9. Constantaras A, Dobbie G, Choromokos E, Frenkel M. Juvenile sex-linked recessive retinoschisis in a black family. Am J Ophthalmol. 1972;74(6):1166-1169, 1171-1178. https://doi.org/10.1016/0002-9394(72)90739-8

10. Ide C, Wilson R. Juvenile retinoschisis. Br J Ophthalmol. 1973;57(8):560-562. https://doi.org/10.1136/bjo.57.8.560 